PAPER

\title{
Counterfactual cognitive deficit in persons with Parkinson's disease
}

P McNamara, R Durso, A Brown, A Lynch

See end of article for authors' affiliations

.....................

Correspondence to: Dr Patrick McNamara, Department of Neurology (127), VA New England Healthcare System, 150 South Huntington Avenue, Boston, MA 02130, USA; mcnamar@bu.edu

Received

8 November 2002

In revised form

23 January 2003

Accepted

4 February 2003
J Neurol Neurosurg Psychiatry 2003;74:1065-1070

Background: Counterfactuals are mental representations of alternatives to past events. Recent research has shown them to be important for other cognitive processes, such as planning, causal reasoning, problem solving, and decision making-all processes independently linked to the frontal lobes. Objective: To test the hypothesis that counterfactual thinking is impaired in some patients with Parkinson's disease and is linked to frontal dysfunction in these patients.

Methods. Measures of counterfactual processing and frontal lobe functioning were administered to 24 persons with Parkinson's disease and 15 age matched healthy controls.

Results. Patients with Parkinson's disease spontaneously generated significantly fewer counterfactuals than controls despite showing no differences from controls on a semantic fluency test; they also performed at chance levels on a counterfactual inference test, while age matched controls performed above chance levels on this test. Performance on both the counterfactual generation and inference tests correlated significantly with performance on two tests traditionally linked to frontal lobe functioning (Stroop colour-word interference and Tower of London planning tasks) and one test of pragmatic social communication skills.

Conclusions: Counterfactual thinking is impaired in Parkinson's disease. This impairment may be related to frontal lobe dysfunction.
C ounterfactuals are mental simulations of "what might have been." They refer to imagined alternatives to something that has actually occurred. They play a significant role in other cognitive processes such as conceptual learning, decision making, social cognition, mood adjustment, and performance improvement. ${ }^{1}$ They help us to process causal relations by highlighting possible causal antecedents of an unpleasant outcome (for example, "if only we had left earlier, we would have avoided the storm"; "if only I had studied harder, I would not have flunked the test"), and to imagine better ways of proceeding ("Henceforth, we will leave earlier ..."; “ . . study harder ...", and so on). They appear to be a pervasive part of normal cognitive processing and may occur fairly often outside of conscious awareness. Without counterfactual thinking a person would find it more difficult to avoid repetition of past mistakes, to adjust their mood after an unpleasant event, to reason effectively about unpleasant events, and so forth.

Knight and Grabowecky ${ }^{2}$ described a man with dorsolateral prefrontal cortical damage in which the most marked behaviour was "a complete absence of counterfactual expressions." This lack of counterfactual expression existed in spite of the fact that the man had recently experienced emotional stressors (for example, a career setback) that are typically associated with the emergence of conscious counterfactual thinking. The man was perseverative and socially impaired as well. More recently, Hooker et al reported that patients with schizophrenia are less likely than controls to mention counterfactual thoughts after recalling personally experienced negative events ${ }^{3}$; in addition, counterfactually derived inferences were reliably different between the schizophrenic subjects and controls, as assessed by scores on a "counterfactual inference test" (CIT) developed by the authors. On the other hand the two groups did not differ on other cognitive measures such as the vocabulary subtest of the WAIS-R, so the counterfactual deficit could not be attributed only to a general cognitive impairment. Like persons with frontal lesions, schizophrenic individuals are perseverative, socially inappropriate, and less efficient learners when compared with healthy people. As many patients with schizophrenia typically show prefrontal lobe dysfunction, the authors suggested that the poor counterfactual performance might be related to that feature. Unfortunately, no data were reported on the strength of relation between performance on tests of frontal function and performance on counterfactual tasks, so no direct evidence of involvement of the frontal lobes was obtained.

Parkinson's disease is characterised by rigidity, bradykinesia, gait disorders, and sometimes tremors. The primary pathology involves loss of dopaminergic cells in the substantia nigra and the ventral tegmental area (VTA). ${ }^{45}$ These two subcortical dopaminergic sites give rise to two projection systems important for motor, affective, and cognitive functioning. The nigrostriatal system, primarily implicated in motor functions, originates in the pars compacta of the substantia nigra and terminates in the striatum. The meso-limbic-cortical system contributes to cognitive and affective functioning. It originates in the VTA and terminates in the ventral striatum, amygdala, frontal lobes, and some other basal forebrain areas. Dopamine levels in the ventral striatum, frontal lobes, and hippocampus are approximately $40 \%$ of normal values in Parkinson's disease. ${ }^{4-7}$ The degree of nigro-striatal impairment correlates with the degree of motor impairment in affected individuals, while VTA-mesocortical dopaminergic impairment correlates positively with the degree of affective and intellectual impairment. ${ }^{8-10}$ The mesocortical dopaminergic dysfunction very probably has a negative impact on prefrontal lobe functions. There is a substantial body of evidence for prefrontal dysfunction in many patients with Parkinson's disease (for reviews, see ${ }^{11-19}$ ) but counterfactual processing has, to our knowledge, never been studied in these patients. Specific deficits that have been linked to prefrontal dysfunction in Parkinson's disease patients include poor planning, perseveration,

Abbreviations: CIT, counterfactual inference test; DSM-III, Diagnostic and Statistical Manual of Mental Disorders, 3rd ed; MMSE, mini-mental state examination; TAS, total achievement score; TOL, Tower of London task; VTA, ventral tegmental area; WAIS, Wechsler adult intelligence scale 
Table 1 The counterfactual inference test (Hooker et al, 2000 ${ }^{3}$ )

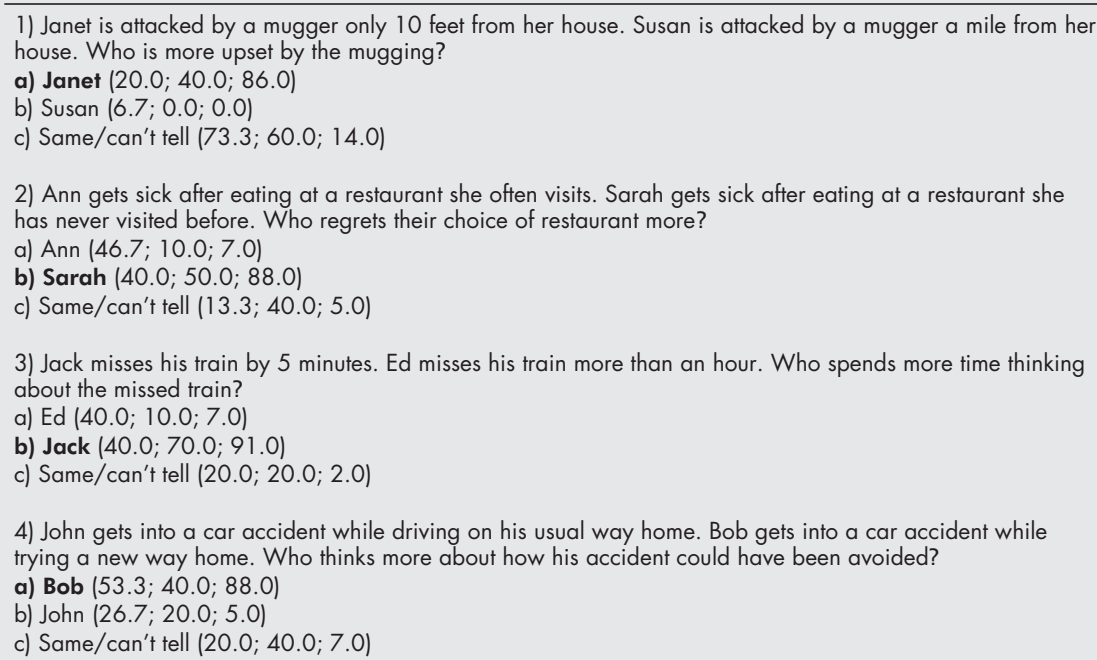

Note: Correct or normative responses to these questions are in bold and are 1) $a, 2) b, 3) b, 4$ ) a.

Numbers in parentheses: \% of Parkinson's disease patients who selected this item; \% age matched controls;

$\%$ undergraduates in Hooker et al pilot validating study who selected this item.

poor problem solving, difficulty generating novel ideas, impaired working memory, and speech monitoring deficits. ${ }^{19} 20$

In this study we attempted to document counterfactual processing abilities in patients with Parkinson's disease, and to examine more directly the relation between counterfactuals and prefrontal function. We administered both counterfactual tests and tests of prefrontal function to a group of patients with Parkinson's disease. If counterfactual thinking depends crucially on intact prefrontal function then it should be impaired in such individuals, who are known to have prefrontal deficits.

\section{METHODS \\ Participants}

Twenty four patients with Parkinson's disease were recruited from the outpatient movement disorders clinic at the VA New England Healthcare System, Boston, Massachusetts. Patients were individually diagnosed by Dr Raymon Durso, director of the clinic. All were male and right handed. The mean disease duration was 8.0 years. None of the patients was demented according to clinical examination and DSM-III criteria. All were on some form of dopaminergic drug treatment and were tested while on treatment. Patients with a history of substance abuse or head injury were excluded.

Fifteen age matched control participants were recruited from the support staff of the same facility and the Boston area community. There was no significant difference in education between the Parkinson's disease patients (mean 12.8 years) and the controls (mean 13.3 years) $(t<1)$, or in age (Parkinson's disease, $70.2(10.4)$ years (mean (SD)); controls, 67.3 (7.9) years; $t(37)=-0.918, \mathrm{p}=0.364)$.

\section{Measures and procedure}

All participants completed a battery of tests that included two counterfactual measures, two measures of general cognitive ability, three measures of prefrontal function, and one measure of social functioning.

\section{Counterfactual tests}

Following Hooker et al, we used two measures of counterfactual thinking: one was a direct measure of the participants' ability to generate counterfactuals derived from their own recent past; the second was a measure of the resultant inferences and insights that emerge from successful manipulation of counterfactual ideas.

The first counterfactual measure focused on the frequency of counterfactual thinking, centring on personal real life events. Participants were first asked to recall a negative personal event; they were given three minutes to consider this event in detail (negative as opposed to positive events were used because past research shows that spontaneous counterfactual thinking is more likely for such events; see Roese and Hur, $\left.1997^{21}\right)$. Participants were then asked explicitly if, as they recalled their personal life event, they had any thoughts of how things might have gone differently-thoughts of "if only's" or "what if's." Responses were tape recorded and the number of discrete counterfactual thoughts (defined in terms of semantically separate antecedents-that is, different alternative actions that might have been taken) was tabulated. This direct solicitation of counterfactual thinking based on retrospective self report has been widely used in research among normal populations. ${ }^{1}$

For the second counterfactual measure we used Hooker et al's counterfactual inference test $(\mathrm{CIT})^{3}$ which is centred on inferences resulting from counterfactual thinking. The CIT is a four question forced choice test based on past research regarding factors that enhance counterfactual thinking. This research had shown first, that outcomes preceded by unusual rather than typical actions heighten counterfactual thinking, ${ }^{22}$ and second, that events that seem "almost" (either spatially or temporally) to have occurred also heighten counterfactual thinking. ${ }^{22}$ For each of the four questions, events experienced by two individuals are presented, and three response options are given. Based on Hooker et al's pilot testing of the instrument, the responses showing normal manipulation of counterfactuals for each of the four questions are la, 2b, 3b, and 4a. When Hooker et al piloted the instrument with a group of undergraduates, the mean CIT score was 3.53 out of a total possible score of 4 . If responses to the CIT were selected randomly, the expected score would be 1.32 (one in three chance multiplied by four items) (see table 1).

\section{General cognitive measures}

The animals category verbal fluency test ${ }^{23}$ was used as a measure of semantic and verbal production. Participants are asked to generate as many names of animals as they can in the space of 
one minute. The mean number of words produced per minute is the outcome measure. The mini-mental state examination $(\text { MMSE })^{24}$ is a short bedside examination that assesses basic orientation to time and place, receptive language abilities, visuo-constructional abilities, and attention as determined by simple serial calculations or spelling of a word backwards. Higher scores (total possible correct $=30$ ) indicate intact cognitive abilities. These control measures were used to confirm that dysfunction in counterfactual thinking was not simply reducible to global deficits in cognition or poverty of speech.

\section{Frontal tests}

The Stroop procedure requires the subject to name the colour of the ink in which a coloured word is printed. The task involves three test cards, one containing rows of coloured rectangles, with the task being to name the colours as quickly as possible, one containing rows of colour words (printed in black ink), with the task being to read the words as quickly as possible, and the third "interference" test consisting of rows of colour words printed in ink colours incongruent with the word represented, with the task being to name the ink colours as quickly as possible. The subject must ignore the word and name the colour. We focus on interference effects in this paper. Susceptibility to cognitive interference is calculated as a ratio of the time taken to name the colours in the incongruent condition. We also tabulated the number of errors committed (for example, giving the word rather than the colour) during the interference condition. Positron emission tomography studies show that orbitofrontal cortex is activated in normal individuals during the interference condition. ${ }^{25}$

In the Tower of London (TOL) task, ${ }^{26}$ disks have to be moved from a starting configuration on three sticks of equal length to a target arrangement in a minimum number of moves. Subjects are asked to rearrange the disks on the sticks so that their positions match the target array (presented as a coloured drawing). The starting position of the discs is varied so that in any particular trial the solution can only be reached following a minimum number of moves. The subject's task is to solve the problem with the minimum number of possible moves. As we were interested in the participant's ability to plan their moves, we used "mean time elapsed until completion of first move" and "mean time per move" as our main outcome measures. We also tabulated the number of problems correctly solved, move accuracy, and the number of rule violations. A SPECT (single photon emission computed tomography) study has shown left prefrontal cortex activation in subjects attempting to plan their moves. ${ }^{27}$

\section{Social functioning}

We focused on social communication skills and used the "pragmatic protocol" developed by Prutting and Kirchner ${ }^{28}$ to assess the appropriateness of social interactions. This is essentially a checklist of common social interactive behaviours that take place during regular conversations: How well does the patient respond to questions for information? Does he provide appropriate amounts of information rather than too much or too little? Does he stay on topic or is he tangential? The protocol is filled out by the examiner during or after 10 to 15 minutes of conversation with the patient. Prutting and Kirchner showed that the test protocol and scoring reaches adequate reliability and validity levels when used with healthy or brain damaged persons. The examiner presents the patient with a series of prescripted open ended questions in an attempt to elicit as much casual conversation as possible. Verbal, paralinguistic aspects, and non-verbal aspects of the patient's conversation are scored as either appropriate or non-appropriate. Points are given for appropriate responses and then converted to percentage inappropriate scores for the following skills: speech acts, maintaining topic, turn taking, lexical selection, style, prosodics and proxemics (maintaining appropriate physical distances with an interlocutor), and so on. Our outcome measure was the percentage of inappropriate scores for each subject. Neuropsychological reviews of social pragmatic communication skills tend to assign a right hemisphere and perhaps a right frontal role in mediating these social pragmatic skills. ${ }^{29}$

\section{RESULTS}

Patients with Parkinson's disease were less likely to generate counterfactual thoughts after recalling a negative memory and in response to direct questions than control participants: $($ mean (SD)) $0.77 \quad(0.97) \quad v \quad 2.07 \quad(1.7) ; t(1,27)=-2.62$, $\mathrm{p}=0.014$. In addition, scores on the CIT were significantly greater for controls than for the Parkinson's disease group, at $2.0(1.2) v 1.17(0.80) ; t(1,27)=-2.12, \mathrm{p}=0.043$. The Parkinson's disease CIT scores were approximately what would be expected if the test options were selected at random (that is, a mean value of 1.32 ).

It can be seen from table 1 (see Parkinson's disease $v$ controls $v$ normative undergraduate per cent response selections in parentheses) that the patients with Parkinson's disease selected their answers in a distinctly atypical pattern relative to both age matched controls and the normative undergraduate responses obtained by Hooker et al in their original validating study. ${ }^{3}$ Items 1 and 3 reflect the fact that counterfactual thinking is enhanced for events that nearly happened compared with events that did not (item 1 is spatial, item 3 is temporal), whereas items 2 and 4 focus on the fact that counterfactual thinking is typically enhanced for unusual rather than normal events. Generally speaking the patients with Parkinson's disease performed most atypically relative to the undergraduate norm, with the age matched controls falling between these two groups. Fewer Parkinson's disease patients chose the correct response on each item (that is, a on $\mathrm{l}$, b on 2 , b on 3 , and a on 4) than either the age matched controls or the undergraduates. Greater percentages of Parkinson's disease patients as compared with both controls and undergraduates chose the "lure" response on all four items (that is, $\mathrm{b}$ on $\mathrm{l}$, a on 2 , a on 3 , and b on 4). While the patients with Parkinson's disease performed atypically on all items, they were most atypical for the "nearly happened" items (land 3) and for those requiring evaluation of affective responses ( 1 and 2: "Who is more upset . . ."; "Who regrets ...more ..."), with very high percentages endorsing the "same/can't tell" response in question 1 and very high percentages selecting the incorrect lure item on question 2. For example, whereas $86 \%$ of the normative sample and $40 \%$ of the age matched controls selected "Janet" as the person who would be most upset, presumably because she was only 10 feet from safety when the mugging occurred (the "nearly happened" response), only $20 \%$ of Parkinson group did so. While only $14 \%$ of the normative undergraduates selected the "don't know - can't tell" response on this item, $73 \%$ of the Parkinson group selected the "don't know" response for the same item. Similarly, only $40 \%$ of Parkinson group selected the normative responses for items 2 and 3, compared with $88 \%$ plus of undergraduates and more than $50 \%$ of controls. The patients with Parkinson's disease approximated the normative response ( $53 \%$ of Parkinson's disease patients $v 88 \%$ of undergraduates) only for question 4 , a question requiring a judgment of "who thinks more about how to prevent a future accident" (in fact they exceeded the percentage of controls $(40 \%)$ in this case). These results indicate that impairment both in counterfactual thinking and in counterfactual derived inferences are associated with Parkinson's disease (tables 1 and 2). Among Parkinson's disease patients, the correlation between the two measures of counterfactual thinking was substantial $(r=0.73, \mathrm{p}=0.01)$.

There were no significant differences between the two groups of participants on the animals category fluency measure of verbal production (controls 18.4 (6.0), Parkinson's disease patients $15.7(5.4) ; t=1.7, \mathrm{p}=0.16)$. Mean correct 
Table 2 Counterfactual thinking, cognitive ability, and prefrontal measures in Parkinson's disease and control participants

\begin{tabular}{llll}
\hline Measure & Parkinson's disease & Control & p Value \\
\hline Counterfactual generation & $0.77(0.97)$ & $2.07(1.7)$ & 0.014 \\
Counterfactual interference test & $1.17(0.80)$ & $2.0(1.2)$ & 0.043 \\
MMSE & $26.78(2.70)$ & $29.20(0.62)$ & 0.001 \\
Animals & $15.7(5.4)$ & $18.4(6.0)$ & 0.16 \\
Pragmatics & 0.20 & 0.08 & 0.01 \\
Stroop & $85.8(19.9)$ & $58.8(11.2)$ & 0.0001 \\
Stroop errors & $3.61(5.60)$ & $0.16(0.38)$ & 0.018 \\
TOL, 1st (s) & $9.14(4.00)$ & $6.86(9.30)$ & 0.405 \\
TOL, T/move (s) & $7.63(10.50)$ & $6.02(3.60)$ & 0.593 \\
TAS & $12.7(5.2)$ & $17.2(4.8)$ & 0.036 \\
\hline
\end{tabular}

Animals, animals category verbal fluency test; MMSE, mini-mental state examination; Pragmatics, Prutting and Kirchner pragmatic protocol social functioning test; Stroop, Stroop colour recognition test; TAS, total achievement score; T/move, time per move; TOL, Tower of London test; 1 st, time to initiation of first move.

scores on the MMSE were significantly different between the two groups (Parkinson's disease 26.78 (2.7), controls 29.2 $(0.62) ; t=-3.7, \mathrm{p}=0.001)$.

With respect to tests of frontal lobe function (table 2), we found that varying numbers of patients with Parkinson's disease (typically two to three patients for any given test/task) could not complete all portions of the tests. Nevertheless, reliable differences between the Parkinson group and the age matched controls emerged on these tasks. On the Stroop interference test we found differences in mean interference completion times for controls $v$ Parkinson's disease patients (58.8 (11.2) $v 85.8$ (19.9) seconds; $t=4.69, \mathrm{p}=0.0001$ ). In addition, accuracy in the Parkinson group, as measured by the number of errors committed, was reduced relative to the age matched controls on this interference task. The mean number of errors for the Parkinson group was 3.61 (5.6) and for the controls, $0.16(0.38) ; t=2.6, \mathrm{p}=0.018$. On the Tower of London task we found no significant differences in mean times for first move between the age matched control group and the Parkinson group (6.86 (9.3) v 9.14 (4.0) seconds, respectively; $\mathrm{p}=0.405)$ or for mean times per move (6.02 (3.6) $v 7.63$ (10.5) seconds; $p=0.593)$. On the other hand, the patients with Parkinson's disease were significantly impaired on this task relative to age matched controls when the number of problems correctly solved, move accuracy, and the number of rule violations were taken into account in a total achievement score (TAS). Mean TAS for Parkinson's disease patients was 12.7 (5.2) and for controls, $17.2(4.8)(t=-2.22, \mathrm{p}=0.036)$. With respect to social communication skills, controls showed a mean inappropriateness score of only $8 \%$, while the Parkinson group was impaired on approximately $20 \%$ of the 30 categories on the pragmatic protocol $(t(1,26)=-2.88, p=0.01)$. These means also appear in table 2 .

Counterfactual thinking was related to social functioningthat is, both the counterfactual generation scores and the CIT scores correlated reliably with the social pragmatic scores, (Bonferroni corrected $r$ values, 0.38 and 0.45 , both $\mathrm{p}<0.05$ ). In addition, the counterfactual generation test scores correlated strongly with TOL initial thinking time scores $(r=0.68$, $\mathrm{p}=0.03)$, and with the Stroop interference time score $(r=-56, \mathrm{p}=0.01)$.

\section{DISCUSSION}

We found that patients with Parkinson's disease were impaired relative to controls on tests of counterfactual thinking and that this impairment was related to poor frontal function rather than to general cognitive impairment. The patients generated fewer counterfactual thoughts in response to recall of an adverse event than the control participants. Also, patients performed at chance levels on a counterfactual inference test where they were required to compute the appropriate counterfactually derived inferences about hypothetical social events. Counterfactual thinking was, however, reliably related to performance on tests of frontal function, correlating significantly with the Tower of London planning task total achievement score and the Stroop interference score.

With respect to the counterfactual generation task (where participants were asked to generate counterfactuals in response to a negative personal event), poor performance by the Parkinson group relative to the control group may have reflected a specific counterfactual generation deficit, a generalised cognitive deficit, or the inability to retrieve or generate as detailed an account of a negative personal event as controls. We think these alternatives to a more specific counterfactual generation deficit are unlikely for several reasons. First, patients with Parkinson's disease and controls did not differ substantially on measures of global cognitive function such as the MMSE and the animals fluency task. The mean Parkinson's disease MMSE score was approximately 27, while the mean for controls was 29 out of a possible total score of 30 . Although the two-point difference was statistically significant it should be kept in mind that MMSE scores above 24 are generally considered to rule out dementia or global cognitive impairment. Thus both groups evidenced no global cognitive impairment. Similarly, both the Parkinson's disease patients and the controls generated roughly equal numbers of items on the animal fluency task, suggesting that semantic retrieval and cognitive response generation abilities were approximately equivalent for the two groups. Finally, each group produced nearly equal numbers of content words per sentence for the counterfactual sentences they generated on this task. The means for the Parkinson group and the control group were 2.5 and 3.8, respectively, and did not differ statistically ( $t$ value less than 1). We therefore tentatively conclude that patients with Parkinson's disease may have a specific deficit relative to age matched controls in counterfactual generation and thinking. We note that further research will be needed before any definite conclusions can be reached about the specificity of the counterfactual deficit in these patients.

While the patients with Parkinson's disease performed most poorly, the elderly controls in our studies did not perform normally (as compared with the undergraduates) on tests of counterfactual thinking. Inspection of patterns of response on the CIT or inference test shows that in terms of choosing correct responses and avoiding lures the controls generally fell between the undergraduates and the Parkinson group. Nevertheless, their overall mean correct score (2.0) on the inference test was not much better than would have been expected on the basis of chance (1.3). In addition, fewer controls chose the correct or normative response for question 4 than did the Parkinson's disease patients.

What, then, accounts for the relatively poor performance of the controls? As mentioned in our introductory review, we believe that counterfactual processing may depend to some 
extent on the neurocognitive functions of the frontal lobes. Frontal lobe dysfunction has been documented in many otherwise healthy elderly individuals. ${ }^{30}$ The mean age of our controls was around 67 years. Many of these elderly subjects performed relatively poorly on both the Stroop and the Tower task (table 2), thus supporting the view that their poor performance on counterfactual tasks may be related to mild frontal dysfunction. We feel that the poor performance of both the Parkinson group and the elderly controls on both the frontal lobe tests and the tasks of counterfactual thinking strongly supports our hypothesis that counterfactual processing depends to some extent on intact frontal systems.

This is the first report, to our knowledge, documenting a counterfactual thinking impairment in patients with Parkinson's disease. We believe that impoverished counterfactual thinking may account for some of the cognitive and personality changes associated with Parkinson's disease. The parkinsonian personality is said to be rigid, stoic, and characterised by low novelty seeking. ${ }^{19}{ }^{20}$ Many patients in the mid to late stages of the disorder are perseverative both on tests of executive cognitive function and in their daily tendency to do counterproductive or even dangerous things (for example, driving a car, working with power tools, taking on complex construction projects), despite warnings to avoid these dangers. We suggest that counterfactual impairment may be one reason why these patients fail to learn from past mistakes and thus why they persist in maladaptive or dangerous behaviours.

Our findings may also carry some relevance for understanding the abilities of Parkinson's disease patients to handle daily social interactions and activities. If patients suffer counterfactual impairment they are less likely to be able to handle social conversations fluently, to formulate plans easily, or to compare alternative outcomes imaginatively, and so forth. The inefficiency in "running mental simulations" will have an impact on fundamental social skills, resulting ultimately in less flexibility and greater withdrawal from social interactions. The counterfactual impairment may also undermine the ability to self monitor, and thus patients do not realise that their communication skills (their ability to stay on topic, to take appropriate turns in a conversation, to provide enough information in a conversation, and so on) need adjustment. Because these kinds of daily social interaction influence the quality of life for the patient, it will be important for future studies to explore the contribution of the counterfactual deficit to daily social interactions with others. Effective coping with a chronic disorder such as Parkinson's disease requires that the patient be able to build up social networks and contacts, not undermine them.

Our data suggest that the root cause of the counterfactual impairment may be linked with frontal dysfunction, as counterfactual performance was correlated with measures of frontal impairment in the patients with Parkinson's disease. Our results support those of Hooker et al, ${ }^{3}$ who found impoverished counterfactual thinking in schizophrenic individuals and speculated that this was caused by prefrontal dysfunction. There are several possible reasons why frontal lobe deficits might yield impairment in counterfactual thinking. To form a counterfactual, one has to hold the memory of an unpleasant event in the working memory stores long enough to compare what actually happened with the counterfactually derived alternative. To hold such complex information in working memory stores requires "resistance to interference," which is a well documented process thought to be mediated by the frontal lobes. ${ }^{31-33}$ Perhaps patients are not able to generate counterfactual models efficiently and to compare these models with memories of the adverse event because of cognitive interference. The correlation between Stroop interference scores and counterfactual performance supports this possibility.

Another possibility involves a faulty a link between affect and cognition. Negative affect directly induces counterfactual thinking, and counterfactual thinking may itself amplify negative affect. ${ }^{1}$ Damasio ${ }^{34}$ suggested that efficient decision making is facilitated by accessing, through orbitofrontal and limbic routes, a "somatic marker" that yields relevant affective information. Patients with frontal dysfunction cannot access this somatic marker and so cannot use such information in decision making processes. Loss of somatic markers, or the inability to retrieve them, would interfere with normal counterfactual processing.

A final possibility concerns the role of the frontal cortex in supporting mental simulations or mental models in working memory of current events or problems. A counterfactual is a simulation of an alternative state of affairs and thus, like other mental models, should be supported in working memory processes of the frontal lobes. The correlation we found between TOL thinking scores and counterfactual performance supports this possibility.

\section{Conclusions}

Counterfactual thinking is fundamental to self reflective thought and a ubiquitous feature of mental life-one that has multifaceted implications for affect, coping, judgments of blame, and expectations for the future (see Roese, $1997^{22}$ for a review). Moreover, published reports indicate that counterfactual thinking may have positive functional benefits on decision making and performance. ${ }^{35}$ Thus counterfactual thinking processes should be targeted for intervention in patients with Parkinson's disease.

\section{ACKNOWLEDGEMENTS}

We thank Neal J Roese of Northwestern University for allowing us to use the CIT (email communication, 01/09/2001). This material is based upon work supported, in part, by the Office of Research and Development, Medical Research Service, Department of Veteran's Affairs, USA.

\section{Authors' affiliations}

P McNamara, R Durso, A Brown, A Lynch, Department of Neurology, Boston University School of Medicine, Boston, Massachusetts, USA

Competing interests: none declared

\section{REFERENCES}

1 Roese NJ, Olson JK. Counterfactual thinking: the intersection of affect and function. In: Zanna KP, ed. Advances in experimental social psychology. San Diego, CA: Academic Press, 1997: 1-59.

2 Knight RT, Grabowecky N. Escape from linear time: prefrontal cortex and conscious experience. In: Gazzaniga NS, ed. The cognitive neurosciences. Cambridge, MA: MIT Press, 1995:1367.

3 Hooker C, Roese NJ, Park S. Impoverished counterfactual thinking is associated with schizophrenia. Psychiatry 2000;63:326-35.

4 Javoy-Agid $F$, Agid $Y$. Is the mesocortical dopaminergic system involved in Parkinson's disease? Neurology 1980;30:1326-30.

5 Agid $Y$, Javoy-Agid M, Ruberg M. Biochemistry of neurotransmitters in Parkinson's disease. In: Fahn CDMS, ed. Movement disorders 2. New York: Butterworth and Co, 1987:166-230.

6 Scatton B, Javoy-Agid F, Rouquier L, et al. Reduction of cortical dopamine, noradrenaline, serotonin, and their metabolites in Parkinson's disease. Brain Res 1983;275:321-8.

7 Shinotoh HC. The use of PET in Parkinson's disease. Brain Cognition 1995;28:297-310

8 Rinne JO, Rummukainen J, Paljarvi L, et al. Dementia in Parkinson's disease is related to neuronal loss in the medial substantia nigra. Ann Neurol 1989;26:47-50.

9 Torack RM, Morris JC. The association of ventral tegmental area histopathology with adult dementia. Arch Neurol 1988;45:497-501.

10 German D, Manaye K, Smith W, et al. Mid-brain dopaminergic cell loss in Parkinson's disease: computer visualization. Ann Neurol 1989;26:507-14

11 Brown R, Marsden C. Cognitive function in Parkinson's disease: from description to theory. Trends Neurosci 1990;13:21-9.

12 Dubois B, Pillon B, Malapani C, et al. Subcortical dementia and Parkinson's disease: what are the cognitive functions of the basal ganglia? In: Wolters E, Scheltens $P$, eds. Mental dysfunction in Parkinson's disease. Amsterdam: ICG, 1995: 195-210.

13 Dubois B, Boller F, Pillon B, et al. Cognitive deficits in Parkinson's disease. In: Grafman FBJ, ed. Handbook of neuropsychology. New York: Elsevier, 1991:195-240. 
14 Gotham A, Brown R, Marsden C. "Frontal" cognitive functioning in patients with Parkinson's disease "on" and "off" levodopa. Brain 1988;11:311-21.

15 Levin $\mathbf{H}$, Eisenberg $\mathrm{H}$, Benton A, eds. Frontal lobe function and dysfunction. Oxford: Oxford University Press, 1991.

16 Wolters E, Scheltens P, ed. Mental dysfunction in Parkinson's disease. Amsterdam: ICG, 1995

17 Taylor A, Saint-Cyr J. Executive function. In: Cummings SHJ, ed. Parkinson's disease: a neurobiological perspective. Oxford: Oxford University Press, 1991.

18 Taylor AE, Saint-Cyr JA, Lang AE. Frontal lobe dysfunction in Parkinson's disease: the cortical focus of neostriatal outflow. Brain 1986; 109:845-83

19 Taylor A, Saint-Cyr J. The neuropsychology of Parkinson's disease. Brain Cognition 1995;28:281-96

20 McNamara P, Obler L, Au R, et al. Speech monitoring skills in Alzheimer's disease, Parkinson's disease and normal aging. Brain Language 1992;42:38-51.

21 Roese NJ, Hur T. Affective determinants of counterfactual thinking. Social Cognition 1997:15:274-90.

22 Roese NJ. Counterfactual thinking. Psychol Bull 1997;1 21:133-43.

23 Lezak ND. Neuropsychological assessment, 3rd ed. New York: Oxford University Press, 1995.

24 Folstein MF, Folstein SE, McHugh PR. "Mini-mental state". A practical method for grading the cognitive state of patients for the clinician. $J$ Psychiatr Res 1975;12:189-98.

25 Bench CJ, Frith CD, Grasby PM, et al. Investigations of the functional anatomy of attention using the Stroop test. Neuropsychologia 1993;31:907-22.
26 Shallice T. Specific impairments in planning. Phil Trans $R$ Soc Lond 1982;289:199-209.

27 Morris RG, Ahmed S, Syed GM, et al. Neural correlates of planning ability: frontal lobe activation during the Tower of London test. Neuropsychologia 1993;31:1367-78.

28 Prutting CA, Kirchner DM. A clinical appraisal of the pragmatic aspects of language. J Speech Hearing Disord 1987;52:105-19.

29 McDonald S. Viewing the brain sideways? Frontal versus right hemisphere explanations of non-aphasic language disorders. Aphasiology 1993;7:535-50.

30 West RL. An application of prefrontal cortex function theory to cognitive aging. Psychol Bull 1996;120:272-92.

31 Goldman-Rakic P. Circuitry of primate prefrontal cortex and regulation of behavior by representational memory. In: Plum VMF, ed. Higher cortical function. Handbook of physiology. New York: American Physiological Society, 1987:373-417.

32 Fuster JM. The prefrontal cortex. Anatomy, physiology and neuropsychology of the frontal lobe, 2nd ed. New York: Raven Press, 1989.

33 Oscar-Berman M, McNamara P, Freedman M. Delayed-response tasks: parallels between experimental ablation studies and findings in patients with frontal lesions. In: Eisenberg HSLHM, ed. Frontal lobe function and injury. New York: Academic Press, 1991:230-55.

34 Damasio AR. Descartes' error: emotion, reason, and the human brain. London: Papermac, 1996.

35 Roese NJ. The functional basis of counterfactual thinking. J Personality Soc Psychol 1994;66:805-18

\section{HISTORICAL NOTE}

\section{A note on hiccups}

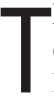
he word hiccup (hiccough or singultus) appears, from its date, to be a variation of the earlier hickock, or hicket. Hiccough, a later spelling, appearing under the erroneous impression that the second syllable was cough; it ought to be abandoned as a mere error (OED).

The English hicket corresponds in formation to the French, and is the earliest form that evolved in series through, hickot, hickock, hickop, to hiccup. For example, T Phaer in Regim Lyfe (1553) remarked,

"It is good to cast colde water in the face of him that hath the hicket."

Many other 16th century writers suggested imaginative domestic remedies. Burton's famous Anatomy of melancholy (1651) notes the effect of shock as a cure:

"By some false accusation, as they do to such as have the hick/hop, to make them forget it."

And in the 1727 Bradley's family dictionary you find:

"You must in the very instant that the hickup seizes the party pull his ring-finger, and it will go off."

The South African hiccup-nut is a fruit of an ornamental shrub, Combretum bracteosum (Poivrea), that presumably affects the hiccups. 20th century medical texts afford little detail. It does not feature in the index of Gowers' first edition but is briefly mentioned on p 295 of the second edition:

"connected with affections of the pneumogastric nerve as they certainly are with the respiratory centre".

SAK Wilson, in Neurology (London, Edward Arnold, 1940; 2nd vol: 1652), provides a brief account without his usual historical notes. He describes a common clonic type and a tonic type seen in tetanus and tonic fits.

Hiccups occur in late fetal life and often in neonates. A hiccup is an intense synchronous contraction of the diaphragmatic and inspiratory intercostal muscles lasting about 500 $\mathrm{ms}$, followed $30 \mathrm{~ms}$ after its onset by glottal closure, which causes the characteristic inspiratory sound and discomfort. It can continue in sleep, and may cease in response to emotional shock. They usually occur with a frequency of $15-30 / \mathrm{min}$. Increasing $\mathrm{PaCO} 2$ and breath holding reduce hiccup frequency (and may eliminate it); but a low PaCO2 increases the amplitude but not frequency. ${ }^{1}$ It seems to be served by a supraspinal mechanism largely distinct from the automatic respiratory system. The characteristics suggest a gastrointestinal nature; its function (if any) is unknown.

Diseases of the medulla in the region of nucleus of tractus solitarius may cause symptomatic hiccup. Common causes are brainstem infarction, tumour, and encephalitis. Uraemia and diverse cardiothoracic, and abdominal pathologies, related to the vagus nerves are well recognised. Many attacks cease spontaneously or respond to pharyngeal stimuli such as drinking iced water. When caused by brainstem or metabolic disease, most methods fail to sustain relief. Raising the PaCO2 by rebreathing from a paper bag is an old but generally effective remedy.

Nasal mucosal stimulation sufficient to provoke sneezing may also abort hiccup, as first reported by Plato in The Symposium, in $416 \mathrm{BC}$ - an observation seldom credited by modern authors.

A related curiosity is high frequency diaphragmatic flutter. Symptoms are of chronic oesophageal belching, hiccups, and, sometimes, dyspnoea, chest pain, and visible epigastric pulsations. EMG and spirometry show 9-15 Hz frequency contractions in the diaphragm, scalene, and parasternal intercostal muscles. Antony van Leeuwenhoek described this condition in himself. ${ }^{2}$ It may respond to carbamazepine. ${ }^{3}$

J M S Pearce 304 Beverley Road, Anlaby, Hull HU10 7BG, UK. jmspearce@freenet.co.uk

\section{References}

1 Newsom-Davis J. An experimental study of hiccup. Brain 1970;93:851-72.

2 Phillips JR, Eldridge FL. Respiratory myoclonus (Leeuwenhoek's disease). N Engl J Med 1973;289:1390-5.

3 Vantrappen G, Decramer M, Harlet R. High-frequency diaphragmatic flutter: symptoms and treatment by carbamazepine. Lancet 1992;339:265-7. 